\title{
Crossover to nearly constant loss in ac conductivity of highly disordered pyrochlore-type ionic conductors
}

\author{
M. R. Díaz-Guillén, ${ }^{1,2}$ J. A. Díaz-Guillén, ${ }^{1}$ A. F. Fuentes, ${ }^{1}$ J. Santamaría, ${ }^{2}$ and C. León ${ }^{2}$ \\ ${ }^{1}$ Cinvestav-Saltillo, Apartado Postal 663, 25000 Saltillo, Coahuila, Mexico \\ ${ }^{2}$ GFMC, Departamento de Física Aplicada III, Facultad de Física, Universidad Complutense de Madrid, Campus Moncloa, 28040 \\ Madrid, Spain
}

(Received 1 February 2010; revised manuscript received 1 October 2010; published 18 November 2010)

\begin{abstract}
We report on ac conductivity measurements of oxide ion conductors with composition $\mathrm{Gd}_{2}\left(\mathrm{Zr}_{y} \mathrm{Ti}_{1-y}\right)_{2} \mathrm{O}_{7}$, at temperatures between 170 and $500 \mathrm{~K}$ and in the frequency range $1 \mathrm{~Hz}-3 \mathrm{MHz}$, and show that a crossover from a sublinear power law to a linear frequency dependence (or nearly constant loss behavior) in the ac conductivity can be clearly observed in a wide temperature range. This crossover is found to be thermally activated, and its activation energy $E_{\mathrm{NCL}}$ to be much lower than the activation energy $E_{\mathrm{dc}}$ for the dc conductivity. We also found that the values of $E_{\mathrm{NCL}}$ are almost independent of composition, and therefore of the concentration of mobile oxygen vacancies, unlike those of $E_{\mathrm{dc}}$. Moreover, for each composition, the values of $E_{\mathrm{NCL}}$ $=0.67 \pm 0.04$ are very similar to those estimated for the energy barrier for the ions to leave their cages, $E_{a}$ $=0.69 \pm 0.05$. These results support that the nearly constant loss behavior, ubiquitous in ionic conductors, is originated from caged ion dynamics.
\end{abstract}

DOI: 10.1103/PhysRevB.82.174304

PACS number(s): $66.30 . \mathrm{H}-$

At enough low temperatures, or high frequencies, ionic conductors show a universal behavior known as nearly constant loss (NCL) ${ }^{1,2}$ It consists of a limiting linear frequency dependence of their ac conductivity,

$$
\sigma_{\mathrm{NCL}}^{\prime}(\omega) \approx A \varepsilon_{0} \omega
$$

where $A$ is a constant with weak temperature dependence ${ }^{3,4}$ and $\varepsilon_{0}$ the permittivity of vacuum. The term "nearly constant loss" is due to the corresponding nearly frequency independent behavior of the dielectric loss $\varepsilon_{\mathrm{NCL}}^{\prime \prime}(\omega)=\sigma_{\mathrm{NCL}}^{\prime}(\omega) / \omega \varepsilon_{0}$ $\approx A$. Despite the great interest in ionically conducting materials during the last decades, most experimental and theoretical work has been devoted to understand the dynamics of mobile ions at much higher temperatures, when the ions eventually give rise to a long-range charge transport characterized by a frequency-independent conductivity $\sigma_{\mathrm{dc}}$, and an NCL contribution to the ac conductivity is usually absent from experimental data. Therefore, until recently, conductivity data showing an NCL behavior were scarce in the literature. ${ }^{5-9}$ It was in 1999 that Ngai first pointed out the correlation existing between the high values of the dc conductivity $\sigma_{\mathrm{dc}}$ and the high values of the NCL magnitude $A$ found in superionic conductors with technological interest for their application as electrolytes in solid-state batteries and fuel cells. ${ }^{4,10}$ Based on this fact and on several other properties of the NCL experimentally observed in ionic conductors, Ngai proposed its physical origin might be due to the displacement of the mobile ions in their local vibrational motion at low temperature and/or high frequency. León et al. ${ }^{11-13}$ later found experimental evidence, in two different lithium ionic conductors, consistent with such an origin for the NCL contribution to the ac conductivity. From the measurement of the crossover temperature (frequency) at which the NCL behavior terminates at different fixed frequencies (temperatures), they were able to determine an activation energy $E_{\mathrm{NCL}}$ for the crossover which was found to be much lower than the activation energy $E_{\mathrm{dc}}$ for the dc conductivity, governed by ion hopping dynamics, and in fact $E_{\mathrm{NCL}}$ was found to be similar to the value estimated for the energy barrier for the ions to leave their cages, $E_{a}$. Different models for ionic conduction in solids have recently explained this experimental finding based on the NCL behavior arising from the cage decay or caged ion dynamics, which result in a logarithmic time dependence of the mean-square displacement and thus in a linear frequency dependence of the ac conductivity. ${ }^{14,15}$ However, there is still some debate on the origin of the NCL in ionic conductors, ${ }^{16-20}$ arising most probably from the scarceness of accurate experimental ac conductivity data of ionic conductors showing a linear frequency dependence in a broad temperature and frequency range, since the dielectric loss value, $A$, and consequently $\tan \delta$ values $\left(\tan \delta=\varepsilon^{\prime \prime} / \varepsilon^{\prime}\right)$ are usually very low in the NCL regime. In particular, it is not easy to obtain enough resolution in measuring the ac conductivity (dielectric loss) values to determine precisely the crossover to the NCL behavior by changing temperature or frequency.

Here we report on ac conductivity data of several oxide ion conductors with composition $\mathrm{Gd}_{2}\left(\mathrm{Zr}_{y} \mathrm{Ti}_{1-y}\right)_{2} \mathrm{O}_{7}$. These materials present a highly disordered pyrochlore structure and have been shown to be promising candidates to substitute fluorites such as yttria-stabilized zirconia as the electrolyte currently used in fuel cells. ${ }^{21,22}$ The cation and anion substructures in the $\mathrm{Gd}_{2}\left(\mathrm{Zr}_{y} \mathrm{Ti}_{1-y}\right)_{2} \mathrm{O}_{7}$ solid solution disorder gradually as the $\mathrm{Zr}$ content increases, leading to the appearance of oxygen vacancies at $48 f$ sites which are known to be responsible for oxygen hopping and diffusion. ${ }^{22,23}$ We show that the crossover to the NCL behavior can be clearly identified in these materials and it is found to be thermally activated with an activation energy $E_{\mathrm{NCL}}$ much lower than the activation energy $E_{\mathrm{dc}}$ for the dc conductivity. We found that the values of $E_{\mathrm{NCL}}$ do not change upon modifying composition unlike those of $E_{\mathrm{dc}}$, and, in fact, $E_{\mathrm{NCL}}$ is similar to the energy barrier of the cages, $E_{a}$, as calculated from computer 
simulations ${ }^{24}$ and also estimated from electrical conductivity relaxation measurements at higher temperatures. ${ }^{25,26}$ This result gives further support to the interpretation of the NCL in ionic conductors coming from the cage decay dynamics at enough low temperature and short times (high frequencies) when the mobile ions still have a very small probability to hop to neighboring vacant sites. ${ }^{14}$ This result highlights also the importance of interactions among mobile ions in determining the values of the activation energy $E_{\mathrm{dc}}$ for the $\mathrm{dc}$ conductivity. ${ }^{27}$

Samples were prepared by mechanically milling stoichiometric mixtures of high-purity elemental oxides, as described in previous works. ${ }^{25}$ Phase composition of milled samples was analyzed by X-ray power diffraction in a Philips X'Pert diffractometer using Ni-filtered $\mathrm{Cu} K \alpha$ radiation $(\lambda$ $=1.5418 \AA$ ) and chemical reactions were considered completed when no traces of the starting reagents were observed by this technique. Impedance spectroscopy measurements were carried out on pellets $(7 \mathrm{~mm}$ diameter and $\sim 0.7 \mathrm{~mm}$ thickness) prepared by uniaxial pressing of the fine powders obtained by milling (sintering temperature $=1500{ }^{\circ} \mathrm{C}$ ). Colloidal Pt paint was coated on both faces to serve as electrodes and measurements were made under $\mathrm{N}_{2}$ gas flow to ensure an inert atmosphere. Impedance data were recorded over the $1 \mathrm{~Hz}-3 \mathrm{MHz}$ frequency range, as a function of temperature, by using a Novocontrol high-resolution alpha analyzer.

Figure 1(a) shows the frequency dependence of the real part of the conductivity at several fixed temperatures for $\mathrm{Gd}_{2} \mathrm{Zr}_{2} \mathrm{O}_{7}$. It can be observed the NCL behavior, i.e., the linear frequency dependence of the conductivity, at the highest frequencies, which becomes more dominant in the experimental frequency window by lowering the temperature. Solid lines in the figure represent the NCL term of the form $\sigma_{\mathrm{NCL}}^{\prime}(\omega)=A \omega \varepsilon_{0}$ for each data set, which helps to identify the crossover frequency at which the NCL behavior ends at each temperature. At lower frequencies, an additional contribution to the conductivity is observed due to ionic hopping, and the conductivity data become sublinearly frequency dependent and eventually become frequency independent in the dc conductivity regime at enough low frequency or high temperature (see conductivity data at $T=190{ }^{\circ} \mathrm{C}$ ). These crossover points are actually better determined by plotting the same data as the frequency dependence of the dielectric loss, $\varepsilon_{\mathrm{NCL}}^{\prime \prime}(\omega)=A$, as in Fig. 1(b), where they are represented by open squares. The criterion used to determine these crossover frequencies $\omega_{x}$ for the end of the NCL was chosen at the point where the dielectric loss is no longer constant when decreasing frequency but takes a value which satisfies the relation $\log \left[\varepsilon^{\prime \prime}\left(\omega_{x}\right) / \varepsilon^{\prime \prime}\left(10 \omega_{x}\right)\right]=0.05$, that is $\varepsilon^{\prime \prime}\left(\omega_{x}\right)$ is already about a $10 \%$ higher than the constant $\varepsilon_{\mathrm{NCL}}^{\prime \prime}(\omega)=A$ value measured at higher frequencies. As observed in Fig. 1(b), choosing this criterion allows enough accuracy in determining the frequency $\omega_{x}$ while it is still very close to the frequency range where NCL behavior is observed. It is important to remark that, as discussed below, choosing different criteria as a $5 \%$ or a $20 \%$ deviation leads to similar conclusions although the values for the crossover frequencies will be slightly different at each temperature.

The temperature dependence of the ac conductivity, for
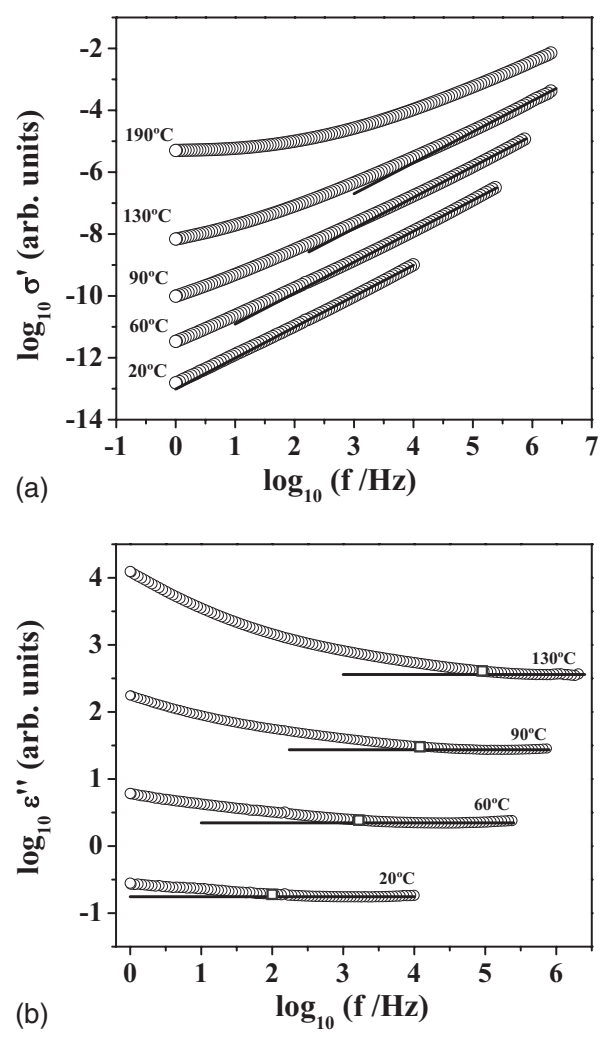

FIG. 1. Frequency dependence of (a) the real part of the conductivity and (b) the imaginary part of the permittivity for $\mathrm{Gd}_{2} \mathrm{Zr}_{2} \mathrm{O}_{7}$ sample at several temperatures. Conductivity data at $T$ $=20{ }^{\circ} \mathrm{C}$ are in siemens per centimeter, and, for clarity, other data sets have been vertically shifted $1,2,3$, and 4 decades, respectively, for $T=60,90,130$, and $190{ }^{\circ} \mathrm{C}$.

the same sample, is represented as an Arrhenius plot in Fig. 2. Each data set is taken at different fixed frequencies, starting at $1 \mathrm{~Hz}$ and up to $1 \mathrm{MHz}$ from the bottom to the top. At the highest temperatures, experimental data for the different frequencies collapse in a single curve, corresponding to the thermally activated dc ionic conductivity values (large open circles). When the temperature is lowered an additional contribution to the conductivity is observed, which is due to the approximately power-law dependence of the ionic ac conductivity, appearing at higher temperature for the highest frequencies. In this regime, ions have already jumped over the potential barriers of their wells but a stationary behavior, i.e., a dc conductivity value, for the long-range ionic transport, has not been achieved yet. And finally, at enough low temperature, we observe the dominant contribution of the NCL to the ac conductivity. Once this regime appears it is found to dominate conductivity curves up to the lowest temperatures. Note that the NCL regime is characterized not only by a linear frequency dependence of ac conductivity but also by a weak temperature dependence, as usually found in ionic conductors. $^{4}$

We have also plotted in Fig. 2 the conductivity values at the corresponding crossover to the NCL behavior (represented by open squares) obtained from the isothermal ac conductivity data as described in Fig. 1. As for the case of the lithium ion conductors analyzed in Ref. 11, the crossover is 


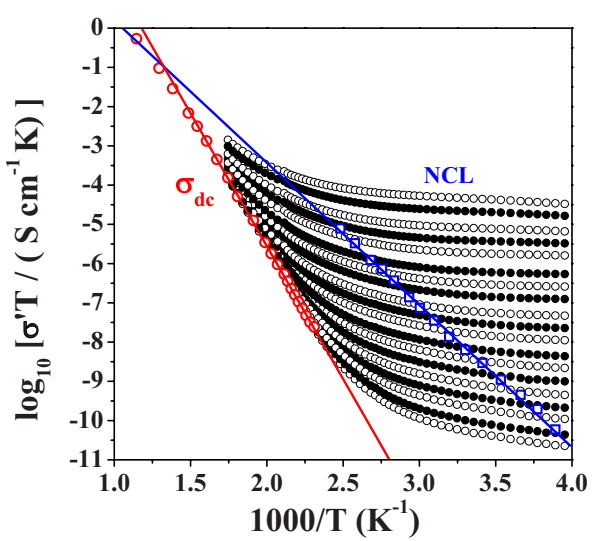

FIG. 2. (Color online) Arrhenius plots showing the temperature dependence of the real part of the conductivity for $\mathrm{Gd}_{2} \mathrm{Zr}_{2} \mathrm{O}_{7}$ sample at several fixed frequencies (from $1 \mathrm{~Hz}$ to $1 \mathrm{MHz}$, three frequencies per decade from bottom to top). Open square symbols represent the crossover to the linear frequency-dependent conductivity or nearly constant loss regime (see text and Fig. 1). Large open circles are dc conductivity data, and the solid lines are fits to an Arrhenius law for dc conductivity data and for the crossover temperature dependence.

found to be thermally activated, and the activation energy $E_{\mathrm{NCL}}=0.71 \pm 0.04 \mathrm{eV}$ is also much lower than that measured for the dc conductivity $\left(E_{\mathrm{dc}}=1.32 \pm 0.04 \mathrm{eV}\right) .{ }^{28}$ This is in fact the expected result for the NCL arising from caged ions with small probability to hop and leave the cages at low temperatures. ${ }^{14,15}$ The lower value of the activation energy would correspond to the actual energy barrier $E_{a}$ that mobile oxygen ions have to overcome to leave their cages and hop into neighboring vacant sites, while the activation energy for long-range ion diffusion $E_{\mathrm{dc}}$ is larger since it takes not only the energy $E_{a}$ but also an additional energy penalty which would be due to the existence of ion-ion interactions and consequently to the existence of unsuccessful ionic hops. According to Ngai's coupling model, ${ }^{29,30}$ it is possible to estimate the energy $E_{a}$ from the frequency-dependent complex conductivity $\sigma^{*}(\omega)$ measurements performed at higher temperatures, where a dc conductivity plateau is observed within the experimental frequency range, by using the relation

$$
E_{a}=(1-n) E_{\mathrm{dc}},
$$

where $n$ is the fractional exponent characterizing the correlation function $\Phi(t)$ of ion hopping, ${ }^{31}$ which is usually well described by a Kohlrausch-Williams-Watts (KWW) function,

$$
\Phi(t)=\exp \left[-(t / \tau)^{1-n}\right] .
$$

This correlation function accounts for the decay of the electric field inside the material under the constrain of a constant displacement vector. ${ }^{31,32}$ Under a constant displacement vector between the electrodes, ions will diffuse inside the material until a concentration gradient is created which progressively cancels the electric field. Thus, the decay of the electric field [and its exact time dependence $\Phi(t)]$ is related to how ions can diffuse inside the material. The use of the electric modulus $M^{*}(\omega)$, which is directly related to the complex conductivity, $M^{*}(\omega)=j \omega \varepsilon_{0} / \sigma^{*}(\omega)$, allows obtaining the

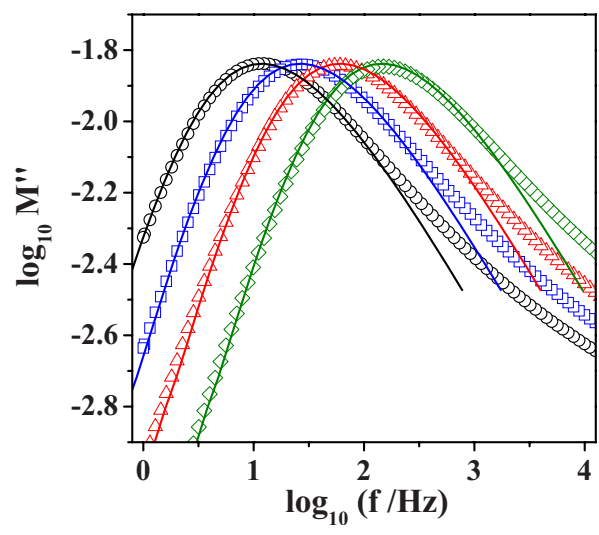

FIG. 3. (Color online) Frequency dependence of the imaginary part of the electric modulus for $\mathrm{Gd}_{2} \mathrm{Zr}_{2} \mathrm{O}_{7}$ sample at temperatures $T=170,180,190$, and $200{ }^{\circ} \mathrm{C}$ from left to right. Solid lines are fits of peaks maxima according to Eqs. (3) and (4).

relaxation function in the time domain, $\Phi(t)$, and determining the value of the exponent $n$ from experimental data. The frequency dependence of the electric modulus is given directly by the Laplace transform of the time derivative of the correlation function $\Phi(t),{ }^{32}$

$$
M^{*}(\omega)=\frac{1}{\varepsilon_{\infty}}\left[1-\int_{0}^{\infty}\left(-\frac{d \Phi}{d t}\right) e^{-j \omega t} d t\right],
$$

where $\varepsilon_{\infty}$ is the dielectric permittivity at high frequencies, and therefore the spectral shape and characteristic time of the electric modulus is determined by the dynamics of mobile ions ${ }^{31,32}$ which we are interested in. In particular, we can analyze the frequency dependence of the experimental electric modulus data in order to determine the value of the exponent $n$ that appears in Eq. (3) by fitting experimental data to Eqs. (3) and (4). According to the coupling model, ${ }^{29,30}$ the exponent $n$ thus obtained relates the activation energy $E_{\tau}$ for the relaxation time $\tau$ in the correlation function to that of a single ion hopping $E_{a}$. Since the activation energy $E_{\mathrm{dc}}$ for the dc conductivity is found to be the same than $E_{\tau}$, it follows that this exponent $n$ obtained from the analysis of the electric modulus data can be used in Eq. (2) which relates the activation energies of conductivity data. It is important also to remark that, despite the controversy that existed in the past about the use of the electric modulus representation, both the electric conductivity and the electric modulus are just different representations of the same experimental data and therefore both of them can be used to analyze ion dynamics. 33,34

The electric modulus data of $\mathrm{Gd}_{2} \mathrm{Zr}_{2} \mathrm{O}_{7}$ at several temperatures are shown in Fig. 3 by $M^{\prime \prime}(\omega)$, the imaginary part of $M^{*}(\omega)$, together with the fits to Eqs. (3) and (4) of the data close to the peak maxima. Note that fits are made by using experimental data up to one decade in frequency higher than the peak frequency since it is well known that deviations from the KWW fit occur at higher frequencies as the NCL regime is approached. ${ }^{34}$ These fits are in nice agreement with the experimental data and a value of the exponent $n=0.44$ was obtained and found to be temperature independent. Thus, by using Eq. (2) and the values determined from ex- 


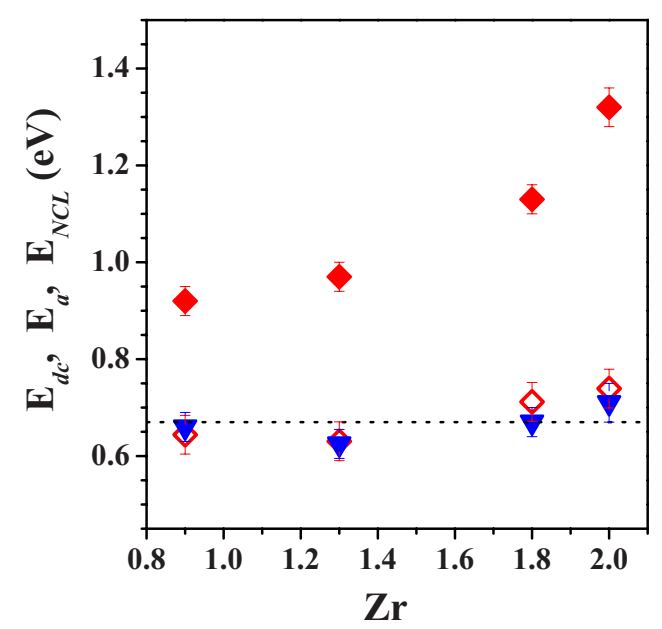

FIG. 4. (Color online) Activation energies $E_{\mathrm{dc}}(\diamond)$ and $E_{a}(\diamond)$ as a function of $\mathrm{Zr}$ content in $\mathrm{Gd}_{2}\left(\mathrm{Zr}_{y} \mathrm{Ti}_{1-y}\right)_{2} \mathrm{O}_{7}$. Dotted line represents the average value $E_{a}=0.67 \mathrm{eV}$ obtained for the energy barrier for oxygen hopping. The activation energy $E_{\mathrm{NCL}}(\boldsymbol{\nabla})$ for the crossover to the nearly constant loss behavior is also plotted, showing similar values to the energy barrier $E_{a}$ for all samples.

periment for $n$ and $E_{\mathrm{dc}}$, a value of the energy barrier $E_{a}$ $=0.74 \pm 0.04 \mathrm{eV}$ is estimated, which is indeed very close, as expected, to the value obtained from Fig. 2 for the energy $E_{\mathrm{NCL}}=0.71 \pm 0.04 \mathrm{eV}$. Moreover, these values are in good agreement with that obtained in these materials from computer simulations for the energy barrier for oxygen ions to hop to neighboring vacant sites. ${ }^{24}$ We want also to emphasize that, as noted above, choosing a different criterion for the determination of the crossover frequencies (as a $5 \%$ or $20 \%$ increase from the NCL value instead of the $10 \%$ value we used) leads to the same value of the activation energy $E_{\mathrm{NCL}}$, which is thus independent of this criterion as far as the crossover frequency is close enough to the NCL regime.

It has been recently reported that by substituting $\mathrm{Ti}$ for $\mathrm{Zr}$ in $\mathrm{Gd}_{2}\left(\mathrm{Zr}_{y} \mathrm{Ti}_{1-y}\right)_{2} \mathrm{O}_{7}$ the activation energy $E_{\mathrm{dc}}$ increases with the $\mathrm{Zr}$ content at temperatures above $200{ }^{\circ} \mathrm{C}$. $^{25,26}$ Interestingly, the exponent $n$ characterizing the KWW time dependence of the corresponding correlation functions was also found to increase with the $\mathrm{Zr}$ content, and in fact the estimated value for the energy barrier $E_{a}$ according to Eq. (2) was obtained to be independent of composition within experimental error. Since it is well known ${ }^{21,35}$ that the number of mobile oxygen vacancies at $48 f$ sites increases by about 2 orders of magnitude by increasing $\mathrm{Zr}$ content from $y=0.3$ to $y=1$, the observed increase in $E_{\mathrm{dc}}$ has been rationalized as just the consequence of the higher value of the exponent $n$ due to an enhanced cooperativity among the mobile oxygen vacancies in the dynamics of the long-range diffusion process, although the microscopic energy $E_{a}$ does not change significantly. ${ }^{25,26}$ Thus, it is also expected that the value of the energy $E_{\mathrm{NCL}}$ remains essentially independent of $\mathrm{Zr}$ content in $\mathrm{Gd}_{2}\left(\mathrm{Zr}_{y} \mathrm{Ti}_{1-y}\right)_{2} \mathrm{O}_{7}$ samples and takes values similar to $E_{a}$ for each composition. In order to check this prediction we have done similar measurements and analysis as those presented for $\mathrm{Gd}_{2} \mathrm{Zr}_{2} \mathrm{O}_{7}$ but for samples with different $\mathrm{Ti} / \mathrm{Zr}$ contents, and the results for $E_{\mathrm{NCL}}$ are shown in Fig. 4. It shows that the activation energy $E_{\mathrm{NCL}}$ is found to be inde-

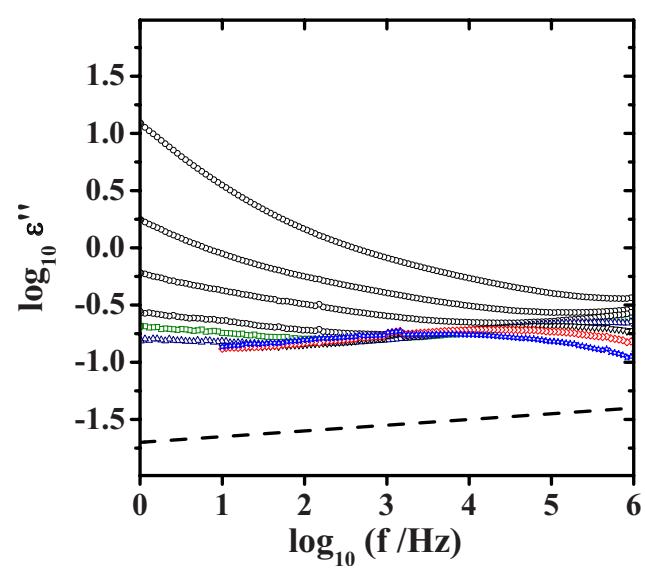

FIG. 5. (Color online) Frequency dependence of the dielectric loss for $\mathrm{Gd}_{2} \mathrm{Zr}_{2} \mathrm{O}_{7}$ at several temperatures: $T=0{ }^{\circ} \mathrm{C}$ (squares), $-20{ }^{\circ} \mathrm{C}$ (up triangles), $-40{ }^{\circ} \mathrm{C}$ (down triangles), $-60{ }^{\circ} \mathrm{C}$ (diamonds), and $-80^{\circ} \mathrm{C}$ (stars). As shown in Fig. 2, all these temperatures are well below the crossover temperature even at the lowest frequency $(1 \mathrm{~Hz})$, and thus the dielectric loss shows the characteristic NCL behavior. Data at higher temperatures are also shown for comparison $\left(T=20,60,90\right.$, and $130{ }^{\circ} \mathrm{C}$, from bottom to top as large open circles). Dashed line represents a weak power-law frequency dependence for the dielectric loss with an exponent of 0.05 and is plotted as a guide to the eyes.

pendent of the $\mathrm{Zr}$ content, as expected, and is in fact in very good agreement with the values for the energy barrier $E_{a}$ obtained by using Eq. (2) with the values of $n$ and $E_{\mathrm{dc}}$ obtained experimentally for each composition.

It is important to emphasize that it is the crossover frequency between the end of the NCL behavior and the onset of ionic hopping response which is found to be thermally activated with activation energy $E_{\mathrm{NCL}}$, and not the amplitude of the NCL itself, which is observed at higher frequencies and shows a very weak temperature dependence (see Fig. 2). Since ion hopping is more strongly dependent on temperature, it dominates the temperature dependence of the crossover frequency and it is found that $E_{\mathrm{NCL}} \approx E_{a}$. In order to further characterize the NCL behavior in these materials, it is necessary to get away from the crossover region and conduct measurements at lower temperatures. Thus we have also performed impedance spectroscopy measurements lowering temperature down to $173 \mathrm{~K}$, that is well below the crossover observed in Fig. 2 even for the lowest frequencies. Figure 5 shows the dielectric loss as a function of frequency for several fixed temperatures in this temperature range together with those measured at higher temperatures. We want to emphasize that once the NCL behavior is reached the dielectric loss becomes almost temperature and frequency independent, as it is in fact expected in ionic conductors. ${ }^{4}$ Note that the maximum slope observed for the frequency dependence of the dielectric loss in the log-log scale of the figure is about 0.05 , i.e., the dielectric loss is changing less than a factor of 2 while the frequency changes over 6 orders of magnitude. One can also readily observe that the dielectric loss shows a very broad peak at the lowest temperatures, which is also a characteristic feature of the NCL behavior in crystalline ionic conductors having a high concentration of mobile ions ${ }^{36}$ like 
in this case. Such a behavior is also reminiscent of the dielectric loss arising from the relaxation of a distribution of asymmetric double-well potentials (ADWPs), a formalism developed by Gilroy and Phillips ${ }^{37}$ which has been previously proposed to account for an NCL contribution originating from the so-called "jellyfish" fluctuations of the atoms in the solid, including the mobile ions. ${ }^{38}$ In the jellyfish concept, a group of atoms collectively moves between different configurations, in contrast with isolated ion hopping determining the conductivity at higher temperatures. Since there is no diffusive, single-ion hopping involved, the ADWP/ jellyfish fluctuations are also possible in the realm of cage dynamics. In fact, recent theoretical work ${ }^{39}$ has shown that the NCL can be understood in terms of a local motion of ions governed by random dipolar interactions between ADWPlike centers which is eventually terminated by thermally activated ion hopping, in agreement with our experimental results.

In summary, we have determined the crossover frequencies at which the NCL behavior ceases to exist in isothermal ac conductivity data of highly disordered pyrochlore-type oxide ion conductors of composition $\mathrm{Gd}_{2}\left(\mathrm{Zr}_{y} \mathrm{Ti}_{1-y}\right)_{2} \mathrm{O}_{7}$ with different $\mathrm{Zr}$ contents. We have found that this crossover is thermally activated with an activation energy similar to that determined for the microscopic energy barrier for mobile oxide ions to hop to neighboring sites, which is found to be significantly lower than the activation energy $E_{\mathrm{dc}}$ for the $\mathrm{dc}$ conductivity that results from long-range diffusion of oxide ions. This result gives further support to the important role played by cooperativity effects among mobile ions, in addition to the energy barrier, in determining the value of the activation energy for the dc conductivity, and is consistent with the NCL in these ionic conductors arising from cage decay dynamics.

This work has been supported by Mexican CONACYT (Grant No. SEP-2003-C02-44075), by Spanish MICINN under Projects No. MAT2008-6517-C02 and CONSOLIDER INGENIO 2010 No. CSD2009-00013 (IMAGINE), and by CAM under PHAMA Grant No. S2009/MAT-1756. M.R.D.-G. thanks CONACYT for a grant to stay at Universidad Complutense.
${ }^{1}$ W. K. Lee, J. F. Liu, and A. S. Nowick, Phys. Rev. Lett. 67, 1559 (1991).

${ }^{2}$ J. Wong and C. A. Angell, Glass Structure by Spectroscopy (Dekker, New York, 1976).

${ }^{3}$ C. H. Hsieh and H. Jain, J. Non-Cryst. Solids 203, 293 (1996).

${ }^{4}$ K. L. Ngai, J. Chem. Phys. 110, 10576 (1999).

${ }^{5}$ C. Cramer, K. Funke, and T. Saatkamp, Philos. Mag. B 71, 701 (1995).

${ }^{6}$ D. L. Sidebottom, P. F. Green, and R. K. Brow, Phys. Rev. Lett. 74, 5068 (1995).

${ }^{7}$ A. S. Nowick, A. V. Vaysleb, and W. Liu, Solid State Ionics $\mathbf{1 0 5}$, 121 (1998)

${ }^{8}$ H. Jain and S. Krishnaswami, Solid State Ionics 105, 129 (1998).

${ }^{9}$ C. León, M. L. Lucía, J. Santamaría, and F. Sánchez-Quesada, Phys. Rev. B 57, 41 (1998).

${ }^{10}$ C. León, K. L. Ngai, and A. Rivera, Phys. Rev. B 69, 134303 (2004).

${ }^{11}$ C. León, A. Rivera, A. Várez, J. Sanz, J. Santamaria, and K. L. Ngai, Phys. Rev. Lett. 86, 1279 (2001).

${ }^{12}$ A. Rivera, C. Leon, C. P. E. Varsamis, G. D. Chryssikos, K. L. Ngai, C. M. Roland, and L. J. Buckley, Phys. Rev. Lett. 88, 125902 (2002).

${ }^{13}$ A. Rivera, C. Leon, J. Sanz, J. Santamaria, C. T. Moynihan, and K. L. Ngai, Phys. Rev. B 65, 224302 (2002).

${ }^{14}$ K. L. Ngai and C. Leon, Phys. Rev. B 66, 064308 (2002).

${ }^{15}$ K. Funke, P. Singh, and R. D. Banhatti, Phys. Chem. Chem. Phys. 9, 5582 (2007).

${ }^{16}$ J. R. Macdonald, Phys. Rev. B 66, 064305 (2002).

${ }^{17}$ W. Dieterich and P. Maass, Chem. Phys. 284, 439 (2002).

${ }^{18}$ B. Roling, C. Martiny, and S. Murugavel, Phys. Rev. Lett. 87, 085901 (2001).

${ }^{19}$ J. Habasaki, K. L. Ngai, and Y. Hiwatari, Phys. Rev. E 66, 021205 (2002).
${ }^{20}$ D. M. Laughman, R. D. Banhatti, and K. Funke, Phys. Chem. Chem. Phys. 11, 3158 (2009).

${ }^{21}$ P. K. Moon and H. L. Tuller, Solid State Ionics 28-30, 470 (1988).

${ }^{22}$ J. Chen, J. Lian, L. M. Wang, R. C. Ewing, R. G. Wang, and W. Pan, Phys. Rev. Lett. 88, 105901 (2002).

${ }^{23}$ P. K. Moon and H. L. Tuller, in Solid State Ionics, MRS Symposia Proceedings No. 135, edited by G. Nazri, R. A. Huggins, and D. F. Shriver (Materials Research Society, Pittsburgh, 1989), p. 149.

${ }^{24}$ R. E. Williford, W. J. Weber, R. Devanathan, and J. D. Gale, J. Electroceram. 3, 409 (1999).

${ }^{25}$ K. J. Moreno, G. Mendoza-Suárez, A. F. Fuentes, J. GarciaBarriocanal, C. Leon, and J. Santamaria, Phys. Rev. B 71, 132301 (2005).

${ }^{26}$ M. R. Díaz-Guillén, K. J. Moreno, J. A. Díaz-Guillén, A. F. Fuentes, K. L. Ngai, J. Garcia-Barriocanal, J. Santamaria, and C. Leon, Phys. Rev. B 78, 104304 (2008).

${ }^{27}$ K. L. Ngai, G. N. Greaves, and C. T. Moynihan, Phys. Rev. Lett. 80, 1018 (1998).

${ }^{28}$ A value of $E_{\mathrm{dc}}=1.13 \mathrm{eV}$ was reported in Ref. 26. The difference with the value found here is due to the different temperature range in both works since there is a slight and gradual decrease in the dc activation energy when the temperature is increased above $300{ }^{\circ} \mathrm{C}$. This behavior has been previously reported in Ref. 27 as a universal feature of ionic conductors.

${ }^{29}$ K. L. Ngai, Comments Solid State Phys. 9, 127 (1979).

${ }^{30}$ K. Y. Tsang and K. L. Ngai, Phys. Rev. E 56, R17 (1997).

${ }^{31}$ C. T. Moynihan, Solid State Ionics 105, 175 (1998).

${ }^{32}$ K. L. Ngai and C. Leon, Phys. Rev. B 60, 9396 (1999).

${ }^{33}$ I. M. Hodge, K. L. Ngai, and C. T. Moynihan, J. Non-Cryst. Solids 351, 104 (2005).

${ }^{34}$ For a review of various representations of conductivity relaxation data see C. T. Moynihan, J. Non-Cryst. Solids 172-174, 
1395 (1994); 203, 359 (1996).

${ }^{35}$ C. Heremans, B. J. Wuensch, J. K. Stalick, and E. Prince, J. Solid State Chem. 117, 108 (1995).

${ }^{36}$ A. S. Nowick and B. S. Lim, Phys. Rev. B 63, 184115 (2001).
${ }^{37}$ K. S. Gilroy and W. A. Phillips, Philos. Mag. B 43, 735 (1981).

${ }^{38}$ H. Jain, S. Krishnaswami, and O. Kanert, J. Non-Cryst. Solids 307-310, 1017 (2002).

${ }^{39}$ W. Dieterich and P. Maass, Solid State Ionics 180, 446 (2009). 